\title{
Inverse result in simultaneous approximation by Baskakov-Durrmeyer-Stancu operators
}

\author{
Vishnu Narayan Mishra ${ }^{1 *}$, Kejal Khatri ${ }^{1}$, Lakshmi Narayan Mishra ${ }^{2,3}$ and Deepmala ${ }^{4}$
}

\section{"Correspondence:}

vishnunarayanmishra@gmail.com ${ }^{1}$ Applied Mathematics and

Humanities Department, Sardar Vallabhbhai National Institute of Technology, Ichchhanath Mahadev Road, Surat, Gujarat 395 007, India Full list of author information is available at the end of the article

\begin{abstract}
In the present paper, we study an inverse result in simultaneous approximation for Baskakov-Durrmeyer-Stancu type operators.

MSC: $41 \mathrm{~A} 25 ; 41 \mathrm{~A} 35 ; 41 \mathrm{~A} 36$

Keywords: hypergeometric series; Baskakov-Durrmeyer-Stancu operators; simultaneous approximation
\end{abstract}

\section{Introduction}

Verma et al. [1] considered Baskakov-Durrmeyer-Stancu (abbr. BDS) operators for $0 \leq$ $\alpha \leq \beta$ as

$$
D_{n, \alpha, \beta}(f, x)=\sum_{k=1}^{\infty} p_{n, k}(x) \int_{0}^{\infty} b_{n, k}(t) f\left(\frac{n t+\alpha}{n+\beta}\right) d t+p_{n, 0}(x) f\left(\frac{\alpha}{n+\beta}\right)
$$

where $b_{n, k}(t)=\frac{1}{B(k+1, n)} \frac{t^{k-1}}{(1+t)^{n+k+1}}$ and $p_{n, k}(x)=\left(\begin{array}{c}n+k-1 \\ k\end{array}\right) \frac{x^{k}}{(1+x)^{n+k}}$.

For $\alpha=\beta=0$, these operators reduce to Baskakov-Durrmeyer operators $D_{n}(f, x)=$ $D_{n, 0,0}(f, x)$. Note that this case was investigated in [2]. Several other researchers have studied in this direction and obtained different approximation properties of many operators, and we mention some of them as [3-8] etc. Verma et al. [1] also studied some approximation properties, asymptotic formula and better estimates for these operators. Recently, Gupta et al. [9] and Mishra and Khatri [10] established point-wise convergence, a Voronovskaja-type asymptotic formula and an error estimate in terms of modulus of continuity of the function and investigated moments of these operators using hypergeometric series, errors estimation in simultaneous approximation, respectively.

Let $C_{v}[0, \infty)$, where $v>0$, be the class of all continuous functions defined on $[0, \infty)$ satisfying the growth condition $|f(t)|=O(1+t)^{\nu}$. The norm $\|\cdot\|_{v}$ on $C_{v}[0, \infty)$ is defined as $\|f\|_{v}=\sup _{0<t<\infty}|f(t)|(1+t)^{-\nu}$.

Let

$$
N_{n, \alpha, \beta}(x, t)=\sum_{k=1}^{\infty} p_{n, k}(x) b_{n, k}(t)+p_{n, 0}(x) \delta\left(\frac{n t+\alpha}{n+\beta}\right),
$$

○2013 Mishra et al:; licensee Springer. This is an Open Access article distributed under the terms of the Creative Commons Attribution License (http://creativecommons.org/licenses/by/2.0), which permits unrestricted use, distribution, and reproduction in any medium, provided the original work is properly cited. 
here $\delta\left(\frac{n t+\alpha}{n+\beta}\right)$ being a type of the Dirac delta function. Then operators (1.1) can be written in the following form:

$$
D_{n, \alpha, \beta}(f, x)=\int_{0}^{\infty} N_{n, \alpha, \beta}(x, t) f\left(\frac{n t+\alpha}{n+\beta}\right) d t .
$$

The operators $D_{n, \alpha, \beta}(f, x)$ are well defined for $f \in C_{v}[0, \infty)$. It is easily checked that the operators $D_{n, \alpha, \beta}$ defined above are linear positive operators and $D_{n, \alpha, \beta}(f, x)=1$. It turns out that the order of approximation by these operators is at best $O\left(n^{-1}\right)$ as $n \rightarrow \infty$, howsoever smooth the function $f$ cab is. Throughout this paper, we denote by $C[a, b]$ the space of all continuous functions on the interval $[a, b]$, the norm $\|\cdot\|_{C[a, b]}$ denotes the sup norm on the space $C[a, b]$. For $f \in C[a, b]$ and a positive integer $k \geq 1$, the $k$ th order modulus of continuity is defined as

$$
\omega_{k}(f, \delta ; a, b)=\sup \left\{\left|\Delta_{h}^{k} f(x)\right|:|h| \leq \delta \text { and } x, x+k h \in[a, b]\right\}
$$

where $\Delta_{h}^{k} f(x)$ is $k$ th forward difference with step length $h$.

A function $f$ is said to belong to the generalized Zygmund class $\operatorname{Liz}(\alpha, k ; a, b)$ if for $\delta>0$ there exists a constant $C$ such that $\omega_{2 k}(f, \delta ; a, b) \leq C \delta^{\alpha k}$. In particular for $k=1$, we simply write $\operatorname{Lip}(\alpha, a, b)$ instead of $\operatorname{Liz}(\alpha, 1 ; a, b)$. By $C_{0}$ we mean the class of continuous functions defined on $(0, \infty)$ having a compact support and $C_{0}^{s}$ the subclass of $C_{0}$, consisting of $s$ times continuously differentiable functions with $\operatorname{supp}\left[a^{\prime}, b^{\prime}\right] \subset(a, b)$ and $[a, b] \subset(0, \infty)$. Also let

$$
G^{(s)}=\left\{g \in C_{0}^{s+2}: \operatorname{supp} g \subset\left[a^{\prime}, b^{\prime}\right]\right\} .
$$

For $f \in C_{0}^{s}$ with $\operatorname{supp} f \subset\left[a^{\prime}, b^{\prime}\right]$, Peetre's $K$-functionals are defined as

$$
K_{s}(\xi, f ; a, b)=\inf _{g \in G^{s}}\left\{\left\|f^{(s)}-g^{(s)}\right\|_{C\left[a^{\prime}, b^{\prime}\right]}+\xi\left(\left\|g^{(s)}\right\|_{C\left[a^{\prime}, b^{\prime}\right]}+\left\|g^{(s+2)}\right\|_{C\left[a^{\prime}, b^{\prime}\right]}\right)\right\}, \quad 0<\xi \leq 1 .
$$

For $0<\alpha<2$ and $f \in C_{0}^{s}$ with $\operatorname{supp} f \subset\left[a^{\prime}, b^{\prime}\right]$, we say that $f \in C_{0}^{s}\left(\alpha, k+1 ; a^{\prime}, b^{\prime}\right)$ if

$$
\left\|f^{(s)}\right\|_{\alpha, s} \equiv \sup _{0<\xi \leq 1} \xi^{-\alpha / 2} K_{s}(\xi, f)<\infty
$$

\section{Auxiliary results}

In the sequel we shall need several lemmas.

Lemma 1 [10] For $n>0, m>0$ and $s \geq 0$, we have

$$
D_{n}\left(t^{s}, x\right)=\frac{\Gamma(n-s+1) \Gamma(s+1)}{\Gamma(n+1)}\left[(1+x)^{s}{ }_{2} F_{1}\left(1-n,-s ; 1 ; \frac{x}{1+x}\right)-(1+x)^{-n}\right] .
$$

Moreover,

$$
D_{n}\left(t^{s}, x\right)=\frac{(n+s-1) !(n-s) !}{n !(n-1) !} x^{s}+\frac{s(s-1)(n+s-2) !(n-s) !}{n !(n-1) !} x^{s-1}+O\left(n^{-m}\right) .
$$


Lemma 2 [10] For $0 \leq \alpha \leq \beta$ and $m>0$, we have

$$
\begin{aligned}
D_{n, \alpha, \beta}\left(t^{s}, x\right) \\
=x^{s} \frac{n^{s}}{(n+\beta)^{s}} \frac{(n+s-1) !(n-s) !}{n !(n-1) !} \\
\quad+x^{s-1}\left\{s(s-1) \frac{n^{s}}{(n+\beta)^{s}} \frac{(n+s-2) !(n-s) !}{n !(n-1) !}+s \alpha \frac{n^{s-1}}{(n+\beta)^{s}} \frac{(n+s-2) !(n-s+1) !}{n !(n-1) !}\right\} \\
\quad+x^{s-2}\left\{s(s-1)^{2} \alpha \frac{n^{s-1}}{(n+\beta)^{s}} \frac{(n+s-3) !(n-s+1) !}{n !(n-1) !}\right. \\
\left.\quad+\frac{s(s-1)}{2} \alpha^{2} \frac{n^{s-2}}{(n+\beta)^{s}} \frac{(n+s-3) !(n-s+2) !}{n !(n-1) !}\right\}+O\left(n^{-m}\right) .
\end{aligned}
$$

Lemma 3 [11] For $m \in \mathbb{N} \cup\{0\}$, if

$$
U_{n, m}(x)=\sum_{k=0}^{\infty} p_{n, k}(x)\left(\frac{k}{n}-x\right)^{m}
$$

then $U_{n, 0}(x)=1, U_{n, 1}(x)=0$, and we have the recurrence relation:

$$
n U_{n, m+1}(x)=x(1+x)\left[U_{n, m}^{\prime}(x)+m U_{n, m-1}(x)\right] .
$$

Consequently, $U_{n, m}(x)=O\left(n^{-[(m+1) / 2]}\right)$, where $[m]$ is an integral part of $m$.

Lemma 4 [1] For $m \in \mathbb{N} \cup\{0\}$, if

$$
\begin{aligned}
\mu_{n, m}(x) & =D_{n, \alpha, \beta}\left((t-x)^{m}, x\right) \\
& =\sum_{k=1}^{\infty} p_{n, k}(x) \int_{0}^{\infty} b_{n, k}(t)\left(\frac{n t+\alpha}{n+\beta}-x\right)^{m} d t+p_{n, 0}(x)\left(\frac{\alpha}{n+\beta}-x\right)^{m},
\end{aligned}
$$

then

$$
\mu_{n, 0}(x)=1, \quad \mu_{n, 1}(x)=\frac{\alpha-\beta x}{n+\beta},
$$

and for $n>m$ we have the recurrence relation:

$$
\begin{aligned}
(n-m)\left(\frac{n+\beta}{n}\right) \mu_{n, m+1}(x)= & x(1+x)\left[\mu_{n, m}^{\prime}(x)+m \mu_{n, m-1}(x)\right] \\
& +\left[(m+n x)+\left(\frac{n+\beta}{n}\right)\left(\frac{\alpha}{n+\beta}-x\right)(n-2 m)\right] \mu_{n, m}(x) \\
& -\left(\frac{\alpha}{n+\beta}-x\right)\left[\left(\frac{\alpha}{n+\beta}-x\right)\left(\frac{n+\beta}{n}\right)-1\right] m \mu_{n, m-1}(x) .
\end{aligned}
$$

From the recurrence relation, it is easily verified that for all $x \in[0, \infty)$, we have

$$
\mu_{n, m}(x)=O\left(n^{-[(m+1) / 2]}\right) .
$$


Lemma 5 [11] There exist polynomials $q_{i, j, s}(x)$ on $[0, \infty)$, independent of $n$ and $k$, such that

$$
x^{s}(1+x)^{s} \frac{d^{s}}{d x^{s}} p_{n, k}(x)=\sum_{\substack{2 i+j \leq s \\ i, j \geq 0}} n^{i}(k-n x)^{j} q_{i, j, s}(x) p_{n, k}(x) .
$$

Lemma 6 Let $0<a<a^{\prime}<a^{\prime \prime}<b^{\prime \prime}<b^{\prime}<b<\infty$ and $f^{(s)} \in C_{0}$ with $\operatorname{supp} f \subset\left[a^{\prime \prime}\right.$, $\left.b^{\prime \prime}\right]$. If

$$
\left\|D_{n, \alpha, \beta}^{(s)}(f, \star)-f^{(s)}\right\|_{C[a, b]}=O\left(n^{-\alpha / 2}\right)
$$

then

$$
K_{s}(\xi, f)=C_{1}\left\{n^{-\alpha / 2}+n \xi K_{s}\left(n^{-1}, f\right)\right\} .
$$

Consequently, $K_{s}(\xi, f) \leq C_{2} \xi^{\alpha / 2}$, i.e., $f \in C_{0}^{s}\left(\alpha, 1 ; a^{\prime}, b^{\prime}\right)$, where $C_{1}$ and $C_{2}$ are some positive constants.

Proof To prove (2.3), it is sufficient to show that

$$
K_{s}(\xi, f) \leq C_{1}\left\{n^{-\alpha / 2}+n \xi K_{s}\left(n^{-1}, f\right)\right\} \quad \text { for all } n \text { sufficiently large. }
$$

Since $\operatorname{supp} f \subset\left[a^{\prime \prime}, b^{\prime \prime}\right]$, therefore by Theorem 2 there exists a function $e^{(i)} \in G^{(s)}$ such that for $i=s$ and $i=s+2$,

$$
\left\|D_{n, \alpha, \beta}^{(i)}(f, \star)-e^{(i)}\right\|_{C[a, b]} \leq C_{3} n^{-1},
$$

which implies that

$$
\begin{aligned}
K_{s}(\xi, f) \leq & 3 C_{3} n^{-1}+\left\|D_{n, \alpha, \beta}^{(s)}(f, \star)-f^{(s)}\right\|_{C\left[a^{\prime}, b^{\prime}\right]} \\
& +\xi\left(\left\|D_{n, \alpha, \beta}^{(s)}(f, \star)\right\|_{C\left[a^{\prime}, b^{\prime}\right]}+\left\|D_{n, \alpha, \beta}^{(s+2)}(f, \star)\right\|_{C\left[a^{\prime}, b^{\prime}\right]}\right) .
\end{aligned}
$$

Thus, it is sufficient to show that there exits a constant $C_{4}$ such that for each $g \in G^{(s)}$,

$$
\left\|D_{h, \alpha, \beta}^{(s+2)}(f, \star)\right\|_{C\left[a^{\prime}, b^{\prime}\right]} \leq C_{4} n\left(\left\|f^{(s)}-g^{(s)}\right\|_{C\left[a^{\prime}, b^{\prime}\right]}+n^{-1}\left\|g^{(s+2)}\right\|_{C\left[a^{\prime}, b^{\prime}\right]}\right) .
$$

In fact, by the linearity property, we have

$$
\left\|D_{n, \alpha, \beta}^{(s+2)}(f, \star)\right\|_{C\left[a^{\prime}, b^{\prime}\right]} \leq\left\|D_{n, \alpha, \beta}^{(s+2)}(f-g, \star)\right\|_{C\left[a^{\prime}, b^{\prime}\right]}+\left\|D_{n, \alpha, \beta}^{(s+2)}(g, \star)\right\|_{C\left[a^{\prime}, b^{\prime}\right]}
$$

Applying Lemma 5, we have

$$
\begin{aligned}
\int_{0}^{\infty}\left|\frac{\partial^{s+2}}{\partial x^{s+2}} N_{n, \alpha, \beta}(x, t)\right| d t \leq & \sum_{\substack{2 i+j \leq s+2 \\
i, j \geq 0}} \sum_{\substack{k=1 \\
\infty, 1}}^{\infty} \frac{n^{i}\left|q_{i, j, s+2}\right|(x)}{[x(1+x)]^{s+2}} p_{n, k}(x)|k-n x|^{j} \int_{0}^{\infty} b_{n, k}(t) d t \\
& +\frac{d^{s+2}}{d x^{s+2}}\left[(1+x)^{-n}\right] .
\end{aligned}
$$


Therefore, by the Cauchy-Schwarz inequality and Lemma 3, we get

$$
\left\|D_{n, \alpha, \beta}^{(s+2)}(f-g, \star)\right\|_{C\left[a^{\prime}, b^{\prime}\right]} \leq C_{5} n\left\|f^{(s)}-g^{(s)}\right\|_{C\left[a^{\prime}, b^{\prime}\right]},
$$

where the constant $N_{4}$ is independent of $f$ and $g$. Next, by Taylor's expansion, we have

$$
g(t)=\sum_{i=0}^{s+1} \frac{g^{(i)}(x)}{i !}(t-x)^{i}+\frac{g^{(s+2)}(\xi)}{(s+2) !}(t-x)^{s+2},
$$

where $\xi$ lies between $t$ and $x$. Using the above expansion and the fact that

$$
\int_{0}^{\infty} \frac{\partial^{s}}{\partial x^{s}} N_{n, \alpha, \beta}(x, t)(t-x)^{i} d t=0 \quad \text { for } s>i
$$

we get

$$
\left\|D_{n, \alpha, \beta}^{(s+2)}(g, \star)\right\|_{C\left[a^{\prime}, b^{\prime}\right]} \leq C_{6}\left\|g^{(s+2)}\right\|_{C\left[a^{\prime}, b^{\prime}\right]}\left\|\int_{0}^{\infty} \frac{\partial^{s+2}}{\partial x^{s+2}} N_{n, \alpha, \beta}(x, t)(t-x)^{s+2} d t\right\|_{C\left[a^{\prime}, b^{\prime}\right]} .
$$

Also, by Lemmas 3, 4 and 5 and the Cauchy-Schwarz inequality, we have

$$
\begin{aligned}
J \equiv & \int_{0}^{\infty}\left|\frac{\partial^{s+2}}{\partial x^{s+2}} N_{n, \alpha, \beta}(x, t)\right|(t-x)^{s+2} d t \\
\leq & \sum_{2 i+j \leq s+2} \sum_{\substack{i, j \geq 0 \\
k=1}}^{\infty} \frac{n^{i}\left|q_{i, j, s+2}\right|(x)}{[x(1+x)]^{s+2}} p_{n, k}(x)|k-n x|^{j} \int_{0}^{\infty} b_{n, k}(t)\left(\frac{n t+\alpha}{n+\beta}-x\right)^{s+2} d t \\
& +\frac{d^{s+2}}{d x^{s+2}}\left[\left(\frac{\alpha}{n+\beta}-x\right)^{s+2}(1+x)^{-n}\right] \\
\leq & \sum_{\substack{2 i+j \leq s+2 \\
i, j \geq 0}} \frac{n^{i}\left|q_{i, j, s+2}\right|(x)}{[x(1+x)]^{s+2}}\left(\sum_{k=1}^{\infty} p_{n, k}(x)(k-n x)^{2 j}\right)^{\frac{1}{2}} \\
& \times\left(\sum_{k=1}^{\infty} p_{n, k}(x) \int_{0}^{\infty} b_{n, k}(t)\left(\frac{n t+\alpha}{n+\beta}-x\right)^{2 s+4} d t\right)^{\frac{1}{2}}\left(\int_{0}^{\infty} b_{n, k}(t) d t\right)^{\frac{1}{2}} \\
& +\frac{d^{s+2}}{d x^{s+2}}\left[\left(\frac{\alpha}{n+\beta}-x\right)^{s+2}(1+x)^{-n}\right] \\
= & C_{7} \sum_{2 i+j \leq s+2} n^{i} O\left(n^{j / 2}\right) O\left(n^{-(s+2) / 2}\right)=C_{8} O(1) .
\end{aligned}
$$

Hence

$$
\left\|D_{n, \alpha, \beta}^{(s+2)}(g, \star)\right\|_{C\left[a^{\prime}, b^{\prime}\right]} \leq C_{9}\left\|g^{(s+2)}\right\|_{C\left[a^{\prime}, b^{\prime}\right]} .
$$

Combining the estimates (2.5)-(2.9), we get (2.4). The other consequence follows from [12]. This completes the proof of the lemma.

Lemma 7 [5] Let $0<a<a^{\prime}<a^{\prime \prime}<b^{\prime \prime}<b^{\prime}<b<\infty$ and $f^{(s)} \in C_{0}$ with $\operatorname{supp} f \subset\left[a^{\prime \prime}, b^{\prime \prime}\right]$. If $\in C_{0}^{s}\left(\alpha, 1 ; a^{\prime}, b^{\prime}\right)$, then $f^{(s)} \in \operatorname{Lip}^{\star}\left(\alpha, a^{\prime}, b^{\prime}\right)$. 


\section{Known and inverse results}

In this section, first we give some known results and then we estimate an inverse theorem in simultaneous approximation for Baskakov-Durrmeyer-Stancu operators. Now, this section is devoted to the following inverse theorem in simultaneous approximation.

Theorem 1 [9] If $s \in \mathbb{N}, f \in C_{v}[0, \infty)$ for some $v>0$, and $f^{(s)}$ exists at a point $x \in(0, \infty)$, then

$$
\lim _{n \rightarrow \infty} D_{n, \alpha, \beta}^{(s)}(f, x)=f^{(s)}(x) .
$$

Theorem 2 [9] Let $f \in C_{v}[0, \infty)$ for some $v>0$, and $f^{(s+2)}$ exists at a point $x \in(0, \infty)$. Then

$$
\begin{aligned}
& \lim _{n \rightarrow \infty} n\left(D_{n, \alpha, \beta}^{(s)}(f, x)-f^{(s)}(x)\right) \\
& \quad=s(s-1-\beta) f^{(s)}(x)+[(2 s-\beta) x+(s+\alpha)] f^{(s+1)}(x)+x(1+x) f^{(s+2)}(x) .
\end{aligned}
$$

Theorem 3 [9] Let $f \in C_{v}[0, \infty)$ for some $v>0$, and $0<a<a_{1}<b_{1}<b<\infty$. Then, for sufficiently large $n$, we have

$$
\left\|D_{n, \alpha, \beta}^{(s)}(f, \star)-f^{(s)}\right\|_{C\left[a_{1}, b_{1}\right]} \leq C_{1} \omega\left(f^{(s)}, n^{-1 / 2}, a, b\right)+C_{2} n^{-k}\|f\|_{\nu},
$$

where $C_{1}=C_{1}(s), C_{2}=C_{2}(s, f)$.

Theorem 4 Let $0<\alpha<2,0<a_{1}<a_{2}<b_{2}<b_{1}<\infty$, and supposef $\in C_{v}[o, \infty)$. Then in the following statements (i) $\Longrightarrow$ (ii):

(i) $\left\|D_{n, \alpha, \beta}^{(s)}(f, \star)\right\|_{C\left[a_{1}, b_{1}\right]}=O\left(n^{-\alpha / 2}\right)$,

(ii) $f^{(s)} \in \operatorname{Lip}^{\star}\left(\alpha, a_{2}, b_{2}\right)$,

where $\operatorname{Lip}^{\star}\left(\alpha, a_{2}, b_{2}\right)$ denotes the Zygmund class satisfying $\omega_{2}\left(f, \delta, a_{2}, b_{2}\right) \leq C \delta^{\alpha}$.

Proof Let us choose $a^{\prime}, a^{\prime \prime}, b^{\prime}, b^{\prime \prime}$ in such a way that $a_{1}<a^{\prime}<a^{\prime \prime}<a_{2}<b_{2}<b<b^{\prime \prime}<b_{1}$. Also suppose $g \in C_{0}^{\infty}$ with supp $g \in\left[a^{\prime \prime}, b^{\prime \prime}\right]$ and $g(x)=1$ on the interval $\left[a_{2}, b_{2}\right]$. For $x \in\left[a^{\prime}, b^{\prime}\right]$ with $D \equiv \frac{d}{d x}$, we have

$$
\begin{aligned}
& D_{n, \alpha, \beta}^{(s)}(f g, x)-(f g)^{(s)}(x) \\
& \quad=D^{s}\left(D_{n, \alpha, \beta}((f g)(t)-(f g)(x)), x\right) \\
& \quad=D^{s}\left(D_{n, \alpha, \beta}(f(t)[g(t)-g(x)], x)\right)+D^{s}\left(D_{n, \alpha, \beta}(g(x)[f(t)-f(x)], x)\right) \\
& \quad=: E_{1}+E_{2} .
\end{aligned}
$$

By the Leibniz formula, we have

$$
\begin{aligned}
E_{1} & =\frac{\partial^{s}}{\partial x^{s}} \int_{0}^{\infty} N_{n, \alpha, \beta}(x, t) f(t)[g(t)-g(x)] d t \\
& =\sum_{i=0}^{s}\left(\begin{array}{l}
s \\
i
\end{array}\right) \int_{0}^{\infty} N_{n, \alpha, \beta}^{(i)}(x, t) \frac{\partial^{s-i}}{\partial x^{s-i}}[f(t)(g(t)-g(x))] d t
\end{aligned}
$$




$$
\begin{aligned}
& =-\sum_{i=0}^{s-1}\left(\begin{array}{c}
s \\
i
\end{array}\right) g^{(s-i)}(x) D_{n, \alpha, \beta}^{(i)}(f, x)+\int_{0}^{\infty} N_{n, \alpha, \beta}^{(s)}(x, t) f(t)(g(t)-g(x)) d t \\
& =: E_{3}+E_{4} .
\end{aligned}
$$

Applying Theorem 3, we have

$$
E_{3}=-\sum_{i=0}^{s-1}\left(\begin{array}{l}
s \\
i
\end{array}\right) g^{(s-i)}(x) f^{(i)}(x)+O\left(n^{-\alpha / 2}\right),
$$

uniformly in $x \in\left[a^{\prime}, b^{\prime}\right]$. By Taylor's expansion of $f(t)$ and $g(t)$, we have

$$
f(t)=\sum_{i=0}^{s} \frac{f^{(i)}(x)}{i !}(t-x)^{i}+O(t-x)^{s}
$$

and

$$
g(t)=\sum_{i=0}^{s+1} \frac{g^{(i)}(x)}{i !}(t-x)^{i}+O(t-x)^{s+1} .
$$

Substituting the above expansions in $E_{4}$ and using Theorem 2, the Schwarz inequality and Lemma 4, we obtain

$$
\begin{aligned}
E_{4} & =\sum_{i=0}^{s} \frac{g^{(i)}(x) f^{(s-i)}(x)}{i !(s-i) !} s !+O\left(n^{-1 / 2}\right) \\
& =\sum_{i=0}^{s}\left(\begin{array}{c}
s \\
i
\end{array}\right) g^{(i)}(x) f^{(s-i)}(x)+O\left(n^{-\alpha / 2}\right),
\end{aligned}
$$

uniformly in $x \in\left[a^{\prime}, b^{\prime}\right]$. Again using the Leibniz formula, we have

$$
\begin{aligned}
E_{2} & =\sum_{i=0}^{s}\left(\begin{array}{c}
s \\
i
\end{array}\right) \int_{0}^{\infty} N_{n, \alpha, \beta}^{(i)}(x, t) \frac{\partial^{s-i}}{\partial x^{s-i}}[g(t)(f(t)-f(x))] d t \\
& =\sum_{i=0}^{s}\left(\begin{array}{l}
s \\
i
\end{array}\right) g^{(s-i)}(x) D_{n, \alpha, \beta}^{(i)}(f, x)-(f g)^{s}(x) \\
& =\sum_{i=0}^{s}\left(\begin{array}{l}
s \\
i
\end{array}\right) g^{(s-i)}(x) f^{(i)}(x)-(f g)^{s}(x)+O\left(n^{-\alpha / 2}\right) \\
& =O\left(n^{-\alpha / 2}\right),
\end{aligned}
$$

uniformly in $x \in\left[a^{\prime}, b^{\prime}\right]$. Combining the above estimates, we get

$$
\left\|D_{n, \alpha, \beta}^{(s)}(f g, \star)-(f g)^{s}\right\|_{C\left[a^{\prime}, b^{\prime}\right]}=O\left(n^{-\alpha / 2}\right) .
$$

Thus by Lemmas 5 and 7 , we have $(f g)^{(s)} \in \operatorname{Lip}^{\star}\left(\alpha, a^{\prime}, b^{\prime}\right)$ also $g(x)=1$ on the interval $\left[a_{2}, b_{2}\right]$, and it proves that $f^{(s)} \in \operatorname{Lip}^{\star}\left(\alpha, a_{2}, b_{2}\right)$. This completes the validity of the implication (i) $\Longrightarrow$ (ii) for the case $0<\alpha \leq 1$.

To prove the result for $1<\alpha<2$ for any interval $\left[a^{*}, b^{*}\right] \subset\left(a_{1}, b_{1}\right)$, let $a_{2}^{*}, b_{2}^{*}$ be such that $\left(a_{2}, b_{2}\right) \subset\left(a_{2}^{*}, b_{2}^{*}\right)$ and $\left(a_{2}^{*}, b_{2}^{*}\right) \subset\left(a_{1}^{*}, b_{1}^{*}\right)$. Letting $\delta>0$ we shall prove the assertion 
$\alpha<2$. From the previous case it implies that $f^{(s)}$ exists and belongs to $\operatorname{Lip}\left(1-\delta, a_{1}^{*}, b_{1}^{*}\right)$. Let $g \in C_{0}^{\infty}$ be such that $g(x)=1$ on the interval $\left[a_{2}, b_{2}\right]$ and $\operatorname{supp} g \subset\left(a_{2}^{*}, b_{2}^{*}\right)$. If $\chi(t)$ denotes the characteristic function of the interval $\left[a_{1}^{*}, b_{1}^{*}\right]$, we have

$$
\begin{aligned}
\left\|D_{n, \alpha, \beta}^{(s)}(f g, x)-(f g)^{(s)}(x)\right\|_{C\left[a_{2}^{*}, b_{2}^{*}\right]} \leq & \left\|D^{s}\left(D_{n, \alpha, \beta}(f(t)[g(t)-g(x)], x)\right)\right\|_{C\left[a_{2}^{*}, b_{2}^{*}\right]} \\
& +\left\|D^{s}\left(D_{n, \alpha, \beta}(g(x)[f(t)-f(x)], x)\right)\right\|_{C\left[a_{2}^{*}, b_{2}^{*}\right]} \\
= & F_{1}+F_{2} .
\end{aligned}
$$

Using the linearity property, the Leibniz formula and Theorem 3, we have

$$
\begin{aligned}
F_{1} & =\left\|D^{s}\left(g(x) D_{n, \alpha, \beta}(f, x)-(f g)(x) D_{n, \alpha, \beta}(1, x)\right)\right\|_{C\left[a_{2}^{*}, b_{2}^{*}\right]} \\
& =\left\|\sum_{i=0}^{s}\left(\begin{array}{l}
s \\
i
\end{array}\right) g^{(s-i)}(x) D_{n, \alpha, \beta}^{(i)}(f, x)-(f g)^{(s)}\right\|_{C\left[a_{2}^{*}, b_{2}^{*}\right]} \\
& =\left\|\sum_{i=0}^{s}\left(\begin{array}{l}
s \\
i
\end{array}\right) g^{(s-i)}(x) f^{(i)}(x)-(f g)^{(s)}\right\| \|_{C\left[a_{2}^{*}, b_{2}^{*}\right]}+O\left(n^{-\alpha / 2}\right)=O\left(n^{-\alpha / 2}\right) .
\end{aligned}
$$

Applying the Leibniz formula and Theorem 2, we get

$$
\begin{aligned}
F_{2} & =\left\|-\sum_{i=0}^{s-1}\left(\begin{array}{l}
s \\
i
\end{array}\right) g^{(s-i)}(x) D_{n, \alpha, \beta}^{(i)}(f, x)+D_{n, \alpha, \beta}^{(s)}(f(t)[g(t)-g(x)] \chi(t), x)\right\|_{C\left[a_{2}^{*}, b_{2}^{*}\right]}+O\left(n^{-1}\right) \\
= & \left\|F_{3}+F_{4}\right\|_{C\left[a_{2}^{*}, b_{2}^{*}\right]}+O\left(n^{-1}\right) .
\end{aligned}
$$

Then by Theorem 3, we have

$$
F_{3}=-\sum_{i=0}^{s-1}\left(\begin{array}{l}
s \\
i
\end{array}\right) g^{(s-i)}(x) f^{(i)}(x)+O\left(n^{-\alpha / 2}\right),
$$

uniformly in $x \in\left[a_{2}^{*}, b_{2}^{*}\right]$. Applying Taylor's expansion of $f(t)$, we have

$$
\begin{aligned}
F_{4}= & \int_{0}^{\infty} N_{n, \alpha, \beta}^{(s)}(x, t)[f(t)(g(t)-g(x)) \chi(t)] d t \\
= & \sum_{i=0}^{s} \frac{f^{(i)}(x)}{i !} \int_{0}^{\infty} N_{n, \alpha, \beta}^{(s)}(x, t)(t-x)^{i}(g(t)-g(x)) \chi(t) d t \\
& +\int_{0}^{\infty} N_{n, \alpha, \beta}^{(s)}(x, t)\left[\frac{f^{(s)}(\xi)-f^{(s)}(x)}{s !}\right](t-x)^{s}(g(t)-g(x)) \chi(t) d t \\
= & : F_{5}+F_{6},
\end{aligned}
$$

where $\xi$ lies between $t$ and $x$. Using Theorem 2, we get

$$
\begin{aligned}
F_{5}= & \sum_{i=0}^{s} \frac{f^{(i)}(x)}{i !} \int_{0}^{\infty} N_{n, \alpha, \beta}^{(s)}(x, t)(t-x)^{i}(g(t)-g(x)) d t+O\left(n^{-1}\right), \\
& \text { uniformly in } x \in\left[a_{2}^{*}, b_{2}^{*}\right] \\
= & F_{7}+O\left(n^{-1}\right) .
\end{aligned}
$$


Again using Taylor's expansion of $g(t) \in C_{0}^{\infty}$ and using the fact that $\varepsilon(t, x) \rightarrow 0$ as $t \rightarrow x$, we have

$$
\begin{aligned}
F_{7}= & \sum_{i=0}^{s} \frac{f^{(i)}(x)}{i !} \int_{0}^{\infty} N_{n, \alpha, \beta}^{(s)}(x, t)(t-x)^{i} \\
& \times\left[g(x)+\sum_{j=1}^{s+2} \frac{g^{(j)}(x)}{j !}(t-x)^{j}+\varepsilon(t, x)(t-x)^{s+2}-g(x)\right] d t \\
= & \sum_{i=0}^{s} \frac{f^{(i)}(x)}{i !} \sum_{j=1}^{s+2} \frac{g^{(j)}(x)}{j !} \int_{0}^{\infty} N_{n, \alpha, \beta}^{(s)}(x, t)(t-x)^{i+j} d t \\
& +\sum_{i=0}^{s} \frac{f^{(i)}(x)}{i !} \int_{0}^{\infty} N_{n, \alpha, \beta}^{(s)}(x, t) \varepsilon(t, x)(t-x)^{i+s+2} d t \\
= & F_{8}+F_{9} .
\end{aligned}
$$

Since $\int_{0}^{\infty} \frac{\partial^{s}}{\partial x^{s}} N_{n, \alpha, \beta}(x, t)(t-x)^{k} d t=0$ for every $s>k$, therefore by Theorem 2 and Lemma 2 , we have

$$
\begin{aligned}
F_{8} & =\sum_{j=1}^{s} \frac{g^{(j)}(x) f^{(s-j)}(x)}{j !(s-j) !} s !+O\left(n^{-1}\right) \\
& =\sum_{j=1}^{s}\left(\begin{array}{l}
s \\
i
\end{array}\right) g^{(j)}(x) f^{(s-j)}(x)+O\left(n^{-1}\right),
\end{aligned}
$$

uniformly in $x \in\left[a_{2}^{*}, b_{2}^{*}\right]$. Also as in the proof of Theorem 1 , it can be easily shown that

$$
F_{9}=O\left(n^{-\alpha / 2}\right)
$$

uniformly in $x \in\left[a_{2}^{*}, b_{2}^{*}\right]$. Next, using Lemma 5 , the mean value theorem, the Schwarz inequality and Lemma 4 , we have

$$
\begin{aligned}
& \left\|\int_{0}^{\infty} N_{n, \alpha, \beta}^{(s)}(x, t)\left[\frac{f^{(s)}(\xi)-f^{(s)}(x)}{s !}\right](t-x)^{s}(g(t)-g(x)) \chi(t) d t\right\|_{C\left[a_{2}^{*}, b_{2}^{*}\right]} \\
& \quad \leq \sum_{\substack{2 i+j \leq s \\
i, j \geq 0}} n^{i+j} \frac{\left|q q_{i, j, s}(x)\right|}{[x(1+x)]^{s}} \\
& \quad \times\left\|\int_{0}^{\infty} N_{n, \alpha, \beta}(x, t)|t-x|^{\delta+s+1} \frac{\left|f^{(s)}(\xi)-f^{(s)}(x)\right|}{s !}\left|g^{\prime}(\eta)\right| \chi(t) d t\right\|_{C\left[a_{2}^{*}, b_{2}^{*}\right]}=O\left(n^{-\delta / 2}\right),
\end{aligned}
$$

where $\eta$ lies between $t$ and $x$, and choose $\delta$ such that $0 \leq \delta \leq 2-\alpha$. Combining the above estimates, we get

$$
\left\|D_{n, \alpha, \beta}^{(s)}(f g, \star)-(f g)^{s}\right\|_{C\left[a_{2}^{*}, b_{2}^{*}\right]}=O\left(n^{-\alpha / 2}\right) .
$$

Since supp $f g \subset\left(a_{2}^{*}, b_{2}^{*}\right)$, therefore by Lemmas 6 and 7 , we have $(f g)^{(s)} \in \operatorname{Lip}^{\star}\left(\alpha, 1, a_{2}^{*}, b_{2}^{*}\right)$ also $g(x)=1$ on the interval $\left[a_{2}, b_{2}\right]$, which proves that $f^{(s)} \in \operatorname{Lip}^{\star}\left(\alpha, a_{2}, b_{2}\right)$. This completes the 
validity of the implication (i) $\Longrightarrow$ (ii) for the case $1<\alpha<2$. This completes the proof of the theorem.

\section{Competing interests}

The authors declare that they have no competing interests.

\section{Authors' contributions}

VNM, KK, LNM and Deepmala computed the auxiliary results and inverse theorem in simultaneous approximation for Baskakov-Durrmeyer-Stancu Operators. VNM and Deepmala conceived of the study and participated in its design and coordination. VNM, KK, LNM and Deepmala contributed equally and significantly in writing this manuscript. All the authors drafted the manuscript, read and approved the final version of manuscript in JIA.

\section{Author details}

${ }^{1}$ Applied Mathematics and Humanities Department, Sardar Vallabhbhai National Institute of Technology, Ichchhanath Mahadev Road, Surat, Gujarat 395 007, India. ${ }^{2}$ L. 1627 Awadh Puri Colony Beniganj, L. 1627 Awadh Puri Colony Beniganj, Phase-III, Opp.-I.T.I., Ayodhya Main Road, Faizabad, Uttar Pradesh 224 001, India. ${ }^{3}$ Department of Mathematics, National Institute of Technology, Silchar, Cachar, Silchar, Assam 788010, India. ${ }^{4}$ School of Studies in Mathematics, Pt. Ravishankar Shukla University, Raipur, Raipur, Chhattisgarh 492010, India.

\section{Authors' information}

Dr. VNM is an assistant professor at the Sardar Vallabhbhai National Institute of Technology, Ichchhanath Mahadev Road, Surat, Gujarat, India and he is a very active researcher in various fields of mathematics like Approximation theory, summability theory, variational inequalities, fixed point theory and applications, operator analysis, nonlinear analysis etc. A Ph.D. in Mathematics, he is a double gold medalist, ranking first in the order of merit in both B.Sc. and M.Sc. Examinations from the Dr. Ram Manohar Lohia Avadh University, Faizabad (Uttar Pradesh), India. Dr. VNM has undergone rigorous training from IIT, Roorkee, Mumbai, Kanpur, ISI Banglore in computer oriented mathematical methods and has experience of teaching post graduate, graduate and engineering students. Dr. VNM has to his credit many research publications in reputed journals including SCI/SCI(Exp.) accredited journals. Dr. VNM is referee of several international journals in the frame of pure and applied mathematics and Editor of reputed journals covering the subject mathematics. The second author KK is a research scholar (R/S) in Applied Mathematics and Humanities Department at the Sardar Vallabhbhai National Institute of Technology, Ichchhanath Mahadev Road, Surat (Gujarat), India under the guidance of Dr. VNM and expert in operator analysis. Recently the third author LNM joined as a full-time research scholar (FIR) at the Department of Mathematics, National Institute of Technology, Silchar-788010, District-Cachar, Assam, India and he is also very good active researcher in approximation theory, summability analysis, integral equations, nonlinear analysis, optimization technique, fixed point theory \& applications and operator theory. The fourth author Deepmala is referee of many journals like as Elsevier Journals, Bull. Math. Anal. Appl. Demonstratio Mathematica, African J. Math. Math. Sci. etc. and she is very expert in Integral Equations, Non-linear analysis, dynamic programming, Fixed point theory and applications etc.

\section{Acknowledgements}

The authors wish to express their gratitude to the anonymous referees for their detailed criticism and elaborate suggestions which have helped them to improve the paper substantially. They have thus been able to eliminate some mistakes and to present the manuscript in a more compact manner. Authors mention their sense of gratitude to their great master and friend academician Prof. Ravi P. Agarwal, Texas A and M University-Kingsville, TX, USA, for kind cooperation, smooth behavior during communication and for his efforts to send the reports of the manuscript timely as well as for supporting this work. The authors are also thankful to all the editorial board members and reviewers of prestigious Science Citation Index (SCI) journal, i.e., Journal of Inequalities and Applications (JIA). This research article is totally supported by CPDA, SVNIT, Surat (Gujarat), India.

Received: 15 July 2013 Accepted: 29 October 2013 Published: 17 Dec 2013

\section{References}

1. Verma, DK, Gupta, V, Agrawal, PN: Some approximation properties of Baskakov-Durrmeyer-Stancu operators. Appl. Math. Comput. 218, 6549-6556 (2012)

2. Finta, Z: On converse approximation theorems. J. Math. Anal. Appl. 312, 159-180 (2005)

3. Agrawal, PN, Thamer, KJ: Approximation of unbounded functions by a new sequence of linear positive operators. J. Math. Anal. Appl. 225, 660-672 (1998)

4. Gupta, V, Noor, MA, Beniwal, MS, Gupta, MK: On simultaneous approximation for certain Baskakov-Durrmeyer type operators. J. Inequal. Pure Appl. Math. 7, Article ID 125 (2006)

5. Gupta, MK, Gupta, V, Kumar, M: Direct and inverse estimates for a new family of linear positive operators. J. Math. Anal. Appl. 330, 799-816 (2007)

6. Govil, NK, Gupta, V: Direct estimates in simultaneous approximation for Durrmeyer type operators. Math. Inequal. Appl. 10, 371-379 (2007)

7. Gupta, V: Some approximation properties on q-Durrmeyer operators. Appl. Math. Comput. 197, 172-178 (2008)

8. Gupta, V, Yadav, R: Direct estimates in simultaneous approximation for BBS operators. Appl. Math. Comput. 218, 11290-11296 (2012)

9. Gupta, V, Verma, DK, Agrawal, PN: Simultaneous approximation by certain Baskakov-Durrmeyer-Stancu operators J. Egypt. Math. Soc. 20(3), 183-187 (2012)

10. Mishra, VN, Khan, HH, Khatri, K, Mishra, LN: Hypergeometric representation for Baskakov-Durrmeyer-Stancu type operators. Bull. Math. Anal. Appl. 5(3), 18-26 (2013) 
11. Gupta, V: A note on modified Baskakov type operators. Approx. Theory Its Appl. 10, 74-78 (1994)

12. Berens, H, Lorentz, GG: Inverse theorem for Bernstein polynomials. Indiana Univ. Math. J. 21, 693-708 (1972)

10.1186/1029-242X-2013-586

Cite this article as: Mishra et al.: Inverse result in simultaneous approximation by Baskakov-Durrmeyer-Stancu operators. Journal of Inequalities and Applications 2013, 2013:586

Submit your manuscript to a SpringerOpen ${ }^{\circ}$ journal and benefit from:

- Convenient online submission

- Rigorous peer review

- Immediate publication on acceptance

Open access: articles freely available online

- High visibility within the field

- Retaining the copyright to your article 